ALEA, Lat. Am. J. Probab. Math. Stat. 14, 719-731 (2017)

\title{
Colonization and Collapse
}

\author{
F. P. Machado, A. Roldán-Correa and R. B. Schinazi \\ Statistics Department, \\ Institute of Mathematics and Statistics, \\ University of São Paulo. \\ CEP 05508-090, São Paulo, SP, Brazil. \\ E-mail address: fmachado@ime.usp.br \\ Instituto de Matemáticas, \\ Universidad de Antioquia. \\ Calle 67, no 53-108, Medellin, Colombia. \\ E-mail address: alejandro.roldan@udea.edu.co \\ Department of Mathematics, \\ University of Colorado, Colorado Springs \\ 80933-7150, Colorado Springs, CO, USA. \\ E-mail address: rschinaz@uccs.edu
}

\begin{abstract}
Many species live in colonies that thrive for a while and then collapse. Upon collapse, very few individuals survive. The survivors start new colonies at other sites that thrive until they collapse, and so on. We introduce spatial and nonspatial stochastic processes for modeling such population dynamic. Besides testing whether dispersion helps survival in a model experiencing large fluctuations, we obtain conditions for the population to get extinct or to survive.
\end{abstract}

\section{Introduction}

A metapopulation model refers to populations that are spatially structured into assemblages of local populations that are connected via migrations. Each local population evolves without spatial structure; it can increase or decrease, survive, get extinct or migrate in different ways. Many biological phenomena may influence the dynamics of a metapopulation; species adopt different strategies to increase its survival probability. See Hanski (1999) for more about metapopulations.

Some metapopulations (such as ants) live in colonies that thrive for a while and then collapse. Upon collapse very few individuals survive. The survivors start new

Received by the editors February 12th, 2016; accepted August 14th, 2017.

2010 Mathematics Subject Classification. 60K35, 60J80, 92D25.

Key words and phrases. Spatial Stochastic Model, Percolation, Metapopulation.

Research supported by CNPq (141046/2013-9 and 310829/2014-3), FAPESP (09/52379-8) and Universidad de Antioquia. 
colonies at other vertices that thrive until they collapse, and so on. In this paper, we introduce stochastic models to model this population dynamic and to test whether dispersion helps survival. Our non-spatial stochastic models are reminiscent of catastrophe models, see Brockwell (1986). However, instead of having the whole population living in a single colony we now have the population dispersed in a random number of colonies. We show that dispersion of the population helps survival, see Section 4. Our spatial model is similar to a contact process, see Liggett (1985), in that individuals give birth to new individuals in neighboring sites. However, in our model there is no limit in the number of individuals per site. Moreover, at collapse time a site loses all its individuals at once. This introduces large fluctuations that are non standard in interacting particle systems and complicate the analysis, see Section 5.

This paper is divided into five sections. In Section 2 we define a spatial stochastic process for colonization and collapse and present some of its properties. In Section 3 the main results are stated. In Section 4 we introduce a non-spatial version of our model and compare it to other models known in the literature. Finally, in Section 5 we prove the results stated in Section 3.

\section{Spatial model}

We denote by $\mathcal{G}=(V, E)$ a connected non-oriented graph of uniformly bounded degree, where $V:=V(\mathcal{G})$ is the set of vertices of $\mathcal{G}$, and $E:=E(\mathcal{G})$ is the set of edges of $\mathcal{G}$. Vertices are considered neighbors if they belong to a common edge. The degree of a vertex $x \in V$ is the number of edges that have $x$ as an endpoint. We will assume that there is an uniform bound $b$ on the degree of every vertex of the graph $\mathcal{G}$. A graph is $k$-regular if all its vertices have degree $k$. The distance $d(x, y)$ between vertices $x$ and $y$ is the minimal amount of edges that one must pass in order to go from $x$ to $y$. With the usual abuse of notation, by $\mathbb{Z}^{d}$ we mean the graph with the vertex set $\mathbb{Z}^{d}$ and the set of edges $\left\{\left\langle\left(x_{1}, \ldots, x_{d}\right),\left(y_{1}, \ldots, y_{d}\right)\right\rangle\right.$ : $\left.\left|x_{1}-y_{1}\right|+\ldots\left|x_{d}-y_{d}\right|=1\right\}$. Also, $\mathbb{T}^{d}, d \geq 2$, denotes the degree $d+1$ homogeneous tree.

We now give an informal description of our process. It is a continuous time spatial process on the graph $\mathcal{G}$ with parameters $\lambda>0$ and $p \in[0,1]$. At any time, each vertex of $\mathcal{G}$ may be either occupied by a colony or empty. Each colony is started by a single individual. The number of individuals in each colony behaves as a Yule process (i.e. pure birth) with birth rate $\lambda \geq 0$ per individual. To each vertex is associated a Poisson process with rate 1 in such a way that when the exponential time occurs at a vertex occupied by a colony, that colony collapses and its vertex becomes empty. At the time of collapse each individual in the colony survives with a (presumably small) probability $p \in(0,1)$ or dies with probability $1-p$. Each individual that survives tries to found a new colony on one of the nearest-neighbor vertices by first picking a vertex at random, independently of the other survivors. If the chosen vertex is occupied, that individual dies, otherwise the individual founds there a new colony. If more than one survivor tries to occupy the same vertex only one of them succeeds and all the others die. We denote by $C C(\mathcal{G}, \lambda, p)$ the Colonization and Collapse process.

We now give a formal description of the process. The $C C(\mathcal{G}, \lambda, p)$ is a continuoustime Markov process whose state space is $\mathbb{N}^{V}$ and whose evolution (status at time 
$t)$ is denoted by $\eta_{t}$. For a vertex $x \in V, \eta_{t}(x)=i$ means that at the time $t$ there are $i$ individuals at the vertex $x$. We consider $\left|\eta_{t}\right|=\sum_{x \in V} \eta_{t}(x)$.

Next we present a graphical representation of $C C(\mathbb{Z}, \lambda, p)$, the Colonization and Collapse process on $\mathbb{Z}$. The ideas presented here are naturally extended for any graph of uniformly bounded degree.

The graphical representation of $C C(\mathbb{Z}, \lambda, p)$ is a clump of Poisson processes marks on the space-time $\mathbb{Z} \times(0, \infty)$. Each mark indicates a random event. The state space of $C C(\mathbb{Z}, \lambda, p)$ is $\mathbb{N}^{\mathbb{Z}}$. A particular vertex is said to be in state $i$ at time $t$ if it holds a colony of $i$ individuals at time $t$.

First consider a family of independent Poisson Processes

$$
\left\{\left\{\left\{N_{i}^{x}\right\}_{i \geq 1}, C^{x}\right\}: x \in \mathbb{Z}\right\},
$$

where for each $x \in \mathbb{Z}$ and $i \geq 1$, the rates for the Poisson processes $N_{i}^{x}$ and $C^{x}$ are $i \lambda$ and 1 , respectively. The $N_{i}^{x}$ processes will take care of birth events at a vertex $x$ when $x$ hosts a colony of $i$ individuals. The process $C^{x}$ will take care of collapse events at vertex $x$.

Consider now the family of random variables

$$
\left\{\left\{X_{m}^{x, j}, Y_{m}^{x, j}\right\}_{m \geq 1}: x \in \mathbb{Z}, j \geq 1\right\},
$$

where the sequence $\left\{X_{m}^{x, j}, Y_{m}^{x, j}\right\}_{m \geq 1}$ is such that $X_{1}^{x, j}, X_{2}^{x, j}, \ldots$ and $Y_{1}^{x, j}, Y_{2}^{x, j}, \ldots$ are independent random variables uniformly distributed on the interval $[0,1]$. The construction of the process is done as follows.

- At the arrival times of the Poisson process $N_{i}^{x}$, if vertex $x$ is in state $i \geq 1$ then its state is changed to $i+1$.

- At the $j$-th arrival time of the Poisson process $C^{x}$, if vertex $x$ is in state $i \geq 1$, then it is changed to 0 . Moreover, the collapse at $x$ affects its neighbors $x-1$ and $x+1$ in the following way. Let

$$
(K, L)_{i, p}^{x, j}:=\left(\sum_{m=1}^{i} \mathbf{1}\left\{X_{m}^{x, j}<p, Y_{m}^{x, j}<1 / 2\right\}, \sum_{m=1}^{i} \mathbf{1}\left\{X_{m}^{x, j}<p, Y_{m}^{x, j} \geq 1 / 2\right\}\right) .
$$

If $K \geq 1$ and the vertex $x-1$ is in state 0 then its state is changed to 1 . If $L \geq 1$ and the vertex $x+1$ is in state 0 then its state is changed to 1 .

This type of graphical construction goes back to Harris (1972). A difficulty that was raised and solved by Harris is that since there are infinitely many Poisson processes there is no first arrival. This is solved by noting that if $t_{0}>0$ is small enough then the number of vertices interacting with a fixed vertex up to time $t_{0}$ is finite. We now prove this fact.

Let $t_{0}$ be fixed. We define the following random graph on $\mathcal{G}$. Let $x$ be a site in $\mathcal{G}$ and $y$ be a nearest neighbor of $x$. We draw an unoriented arc between $x$ and $y$ if there is at least one collapse at $x$ or at $y$ by time $t_{0}$. We say that two vertices $u$ and $v$ are connected in the random graph if there exists a sequence $y_{0}, y_{1}, \ldots, y_{n}$ of distinct sites such that $y_{0}=u, y_{n}=v,\left(y_{i}, y_{i-1}\right)$ are nearest neighbors and there is an arc between $y_{i}$ and $y_{i-1}$ for each $i=1,2, \ldots, n$. Let $O$ be a fixed site in $\mathcal{G}$. Let $N(n)$ be the number of sites that are connected to $O$ by a path of length $n$. Then,

$$
E(N(n)) \leq b(b-1)^{n-1}\left(1-e^{-2 b t_{0}}\right)^{n},
$$

where $b$ bounds the number of degrees per site in $\mathcal{G}$. This is so because there are less than $b(b-1)^{n-1}$ length $n$ self-avoiding paths in $\mathcal{G}$ that start at $O$. Moreover, the probability that an arc between two neighbors be present is less than $1-e^{-2 b t_{0}}$ 
(recall that collapses occur at rate 1 ). We can now pick $t_{0}>0$ small enough so that $E(N(n))$ converges to 0 as $n$ goes to infinity. This shows that the probability that $O$ be connected to infinitely many sites in the random graph is 0 .

This proves that up to time $t_{0}$ all the clusters of interacting vertices are finite. Therefore, we can use the algorithm described in the graphical construction to construct the process on every finite cluster up to time $t_{0}$. We then construct the process from time $t_{0}$ to time $2 t_{0}$ and so on. Observe also that the graphical construction specifies a unique Markov process.

- Observe that the graphical construction allows for the coupling of two processes with different $\lambda$ and $p$. This makes it easy to show that the process is monotone in $p$ and $\lambda$.

Definition 2.1. Let $\eta_{t}$ be a $C C(\mathcal{G}, \lambda, p)$, starting with a finite number of colonies. If $\mathbb{P}\left(\left|\eta_{t}\right| \geq 1\right.$ for all $\left.t \geq 0\right)>0$ we say that $\eta_{t}$ survives (globally). Otherwise, we say that $\eta_{t}$ dies out (globally).

Remark 2.2. If the process $\eta_{t}$ starts from an infinite number of colonies, then $\mathbb{P}\left(\left|\eta_{t}\right| \geq 1\right.$ for all $\left.t \geq 0\right)=1$, which means that $\eta_{t}$ survives with probability 1 . Still we can see local death according to the following definition.

Definition 2.3. Let $\eta_{t}$ be a $C C(\mathcal{G}, \lambda, p)$. We say that $\eta_{t}$ dies locally if for any vertex $x \in V$ there is a finite random time $T$ such that $\eta_{t}(x)=0$ for all $t>T$. Otherwise we say that $\eta_{t}$ survives locally.

Remark 2.4. Local death corresponds to a finite number of colonizations for every vertex. It is clear that global death implies local death but the opposite is not always true. As an example consider $\eta_{t}$ a $C C\left(\mathbb{Z}^{d}, 0,1\right)$ with $\left|\eta_{0}\right|=1$. In this case $\eta_{t}$ can be seen as a symmetric random walk on $\mathbb{Z}^{d}$. For $d=1$ or 2 , it is recurrent, which implies that $\eta_{t}$ survives locally. For $d \geq 3$, it is transient, which implies that $\eta_{t}$ dies locally but survives globally.

By coupling arguments one can see that $\mathbb{P}\left(\left|\eta_{t}\right| \geq 1\right.$ for all $\left.t \geq 0\right)$ is a nondecreasing function of $\lambda$ and also of $p$. So we define

$$
\lambda_{c}(p, \mathcal{G}):=\inf \left\{\lambda: \mathbb{P}^{\delta_{x}}\left(\left|\eta_{t}\right| \geq 1 \text { for all } t \geq 0\right)>0\right\},
$$

where $x$ is a fixed vertex, and $\mathbb{P}^{\delta_{x}}$ is the law of the process $\eta_{t}$ starting with one colony at $x$. The function $\lambda_{c}(p, \mathcal{G})$ is non-increasing in $p$. Moreover, $\lambda_{c}(1, \mathcal{G})=0$ and $\lambda_{c}(0, \mathcal{G})=\infty$.

Definition 2.5. Let $\eta_{t}$ be a $C C(\mathcal{G}, \lambda, p)$ with $0<p<1$. We say that $\eta_{t}$ exhibits phase transition (on $\lambda$ ) if $0<\lambda_{c}(p, \mathcal{G})<\infty$.

Remark 2.6. Using coupling arguments, we can construct $\eta_{t}$ and $\hat{\eta}_{t}$ as two copies of $C C(\mathcal{G}, \lambda, p)$ such that $\eta_{t} \leq \hat{\eta}_{t}$ for all times $t>0$, provided that $\eta_{0} \leq \hat{\eta}_{0}$. This monotonic property implies that if $\eta_{t}$ survives, $\hat{\eta}_{t}$ also does. Moreover, if $\hat{\eta}_{t}$ dies out (or dies locally) then $\eta_{t}$ does too.

Observe that, as the number of individuals per vertex is not bounded, it is conceivable that the process survives on a finite graph. Next we show that it does not happen.

Proposition 2.7. For any finite graph and starting from any initial configuration, the colonization and collapse process dies out. 
Proof: Let $\alpha$ be the probability that at a colony collapse time, zero individuals attempts to found new colonies at neighboring vertices.

The probability that a Yule process, starting from one individual, has $j$ individuals at time $t$ is $e^{-\lambda t}\left(1-e^{-\lambda t}\right)^{j-1}$, hence we have that

$$
\alpha=\int_{0}^{\infty} e^{-t} \sum_{j=1}^{\infty} e^{-\lambda t}\left(1-e^{-\lambda t}\right)^{j-1}(1-p)^{j} d t .
$$

Let $n$ and $m$ be the number of vertices of $\mathcal{G}$ and its maximum degree, respectively. In order to show sufficient conditions for extinction we couple the number of colonies in the original model to an auxiliary continuous-time branching process $X_{t}$. Particles in the process $X_{t}$ will play a role analogous to colonies in the colonization and collapse process. We now describe $X_{t}$. Each particle is independently associated to an exponential random variable of rate 1 in such a way that, when its exponential time occurs, it dies with probability $\alpha$ or is replaced by $m$ particles with probability $1-\alpha$. We also consider a restriction that makes the total number of particles always smaller or equal than $n$ by suppressing the births that would make $X_{t}$ larger than $n$.

Let $C_{t}$ be the number of colonies at time $t$ in the colonization and collapse process. At time $t=0$ let $X_{0}=C_{0}$. Moreover, we couple each particle in $X_{0}$ to a colony in the colonization and collapse process by using the same exponential random variable of rate 1 . When an exponential occurs there are two possibilities. With probability $\alpha$, both $X_{t}$ and $C_{t}$ decrease by 1 . With probability $1-\alpha$, the process $X_{t}$ grows by $m-1$ particles and $C_{t}$ grows by at most $m-1$ colonies. This is so because in the colonization process we have spatial constraints and attempted colonizations only occur at vertices that are empty. Moreover, a colony always starts with a single individual. Hence, new colonies correspond to births for $X_{t}$. We couple each new colony to a new particle in $X_{t}$ by using the same mean 1 exponential random variable. This coupling yields for all $t \geq 0$

$$
X_{t} \geq C_{t} .
$$

Note now that $\alpha>0$ and that $X_{t}$ is a finite Markov process with an absorbing state. Hence, $X_{t}$ dies out with probability 1 . So does $C_{t}$ and therefore the colonization and collapse process.

\section{Main Results}

Next, we show sufficient conditions for global extinction and local extinction for the colonization and collapse process on infinite graphs.

Theorem 3.1. Let $\mathcal{G}$ be an m-regular graph, $\eta_{t}$ a $C C(\mathcal{G}, \lambda, p)$ and

$$
\mu(m)=m-\frac{m}{\lambda} \sum_{k \geq 1} B\left(1+\frac{1}{\lambda}, k\right)\left(1-\frac{p}{m}\right)^{k},
$$

where $B(a, b)=\int_{0}^{1} u^{a-1}(1-u)^{b-1} d u$ is the beta function.

(i) If $\mu(m) \leq 1$ and $\left|\eta_{0}\right|<\infty$, then $\eta_{t}$ dies out locally and globally.

(ii) Let $\mathcal{G}=\mathbb{Z}^{d}, d \geq 1$. If $\mu(2 d)<1$ and $\eta_{0}(x) \leq 1$ for every $x$ in $\mathbb{Z}^{d}$ then $\eta_{t}$ dies locally.

(iii) Let $\mathcal{G}=\mathbb{T}^{d}, d \geq 1$. If $\mu(d+1)<1 / d$ and $\eta_{0}(x) \leq 1$ for every $x$ in $\mathbb{T}^{d}$ then $\eta_{t}$ dies locally. 
Remark 3.2. Observe that for all $m \geq 1$ and $\lambda$ fixed, there exists $p>0$ such that $\mu(m) \leq 1$. Furthermore, $\mu(m)$ can be expressed in terms of the Gauss hypergeometric function ${ }_{2} F_{1}$ (see Luke (1969)),

$$
\mu(m)=m-\frac{m-p}{\lambda+1}{ }_{2} F_{1}\left(1,1 ; 2+\frac{1}{\lambda} ; 1-\frac{p}{m}\right) .
$$

Next, we show sufficient conditions for survival for colonization and collapse process on some infinite graphs.

Theorem 3.3. For $p>0$ and $\lambda:=\lambda(p, \mathcal{G})>0$ large enough, the $C C(\mathcal{G}, \lambda, p)$ with $\mathcal{G}=\mathbb{Z}^{d}$ or $\mathbb{T}^{d}$ survives globally and locally.

Remark 3.4. From Theorems 3.1 and 3.3 it follows that for $\mathcal{G}=\mathbb{Z}^{d}$ or $\mathbb{T}^{d}$ and $p \in(0,1)$, there exists phase transition (on $\lambda)$ for $C C(\mathcal{G}, \lambda, p)$. So, there exists a non-increasing function $\lambda_{c}(\cdot, \mathcal{G}):(0,1) \rightarrow \mathbb{R}^{+}$such that the survival and extinction regime for $C C(\mathcal{G}, \lambda, p)$ can be schematically represented as in Figure 3.1. In fact $\lambda_{c}(0, \mathcal{G})=\infty$ and $\lambda_{c}(1, \mathcal{G})=0$ but we do not know if $\lambda_{c}(p, \mathcal{G})$ is continuous and strict monotonic.

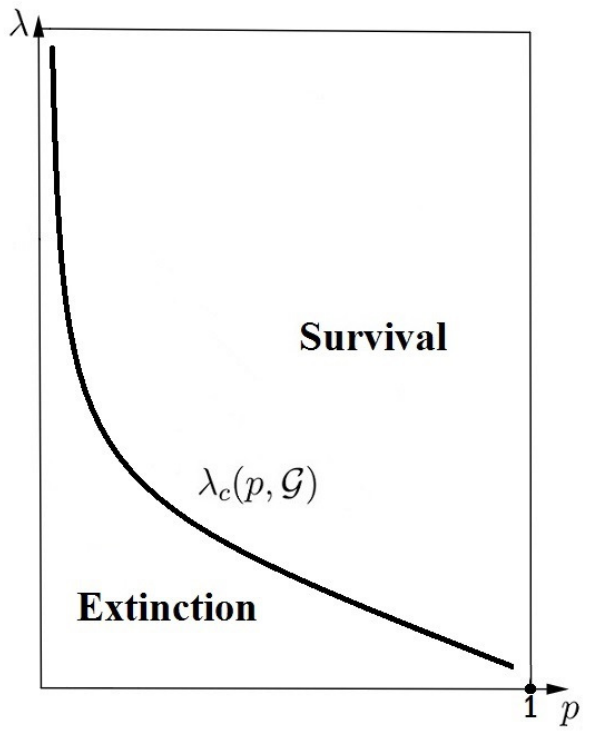

Figure 3.1. Phase transition to $C C(\mathcal{G}, \lambda, p)$, with $\mathcal{G}=\mathbb{Z}^{d}\left(\right.$ or $\left.\mathbb{T}^{d}\right)$.

\section{Non spatial models}

So called Catastrophe Models have been studied extensively and are quite close to our model, see Kapodistria et al. (2016) for references on the subject. Particularly relevant is the birth and death process with binomial catastrophes, see Example 2 in Brockwell (1986). We now describe this model. It is a single colony model. Each individual gives birth at rate $\lambda>0$ and dies at rate $\mu>0$. Moreover, catastrophes (i.e. collapses) happen at rate $a>0$. When a catastrophe happens, every individual 
in the colony has a probability $p$ of surviving and $1-p$ of dying, independently of each other. Brockwell (1986) has shown that survival (i.e. at all times there is at least one individual in the colony) has positive probability if and only

$$
\mu-a \log p<\lambda
$$

Hence, there is a critical value for $p$,

$$
p_{1}=\exp \left(-\frac{\lambda-\mu}{a}\right)
$$

The single colony model survives if and only if $p>p_{1}$.

Next we introduce a non-spatial version of our model and compare it to the catastrophe model above. Consider a model for which every individual gives birth at rate $\lambda$ and dies at rate $\mu$. We start with a single individual and hence with a single colony. When a colony collapses, individuals in the colony survive with probability $p$ and die with probability $1-p$ independently of each other. Every surviving individual founds a new colony which eventually collapses. Colonies collapse independently of each other at rate $a>0$. The proof in Schinazi (2015) may be adapted to show that survival has positive probability if and only if

$$
p E[\exp ((\lambda-\mu) T)]>1,
$$

where $T$ has a rate $a$ exponential distribution. It is easy to see that if $\lambda \geq \mu+a$ then the expected value on the l.h.s. is $+\infty$ and the inequality holds for any $p>0$. It is also easy to see that the inequality cannot hold if $\lambda \leq \mu$. Hence, from now on we assume that $\mu<\lambda<\mu+a$. After computing the expected value and solving for $p$ we get that survival is possible if and only if

$$
p>1-\frac{\lambda-\mu}{a} .
$$

That is, when $\mu<\lambda<\mu+a$ the model with multiple colonies has a critical value

$$
p_{2}=1-\frac{\lambda-\mu}{a} \text {. }
$$

The multiple colonies model survives if and only if $p>p_{2}$.

Since $\exp (-x)>1-x$ for all $x \neq 0$ we have that $p_{1}>p_{2}$ for any $\lambda>\mu$. Hence, it is easier for the model with multiple colonies to survive than it is for the model with a single colony. That is, living in multiple smaller colonies is a better survival strategy than living in a single big colony. Note that this conclusion was not obvious. The one colony model has a catastrophe rate of $a$ while the multiple colonies model has a catastrophe rate of $n a$ if there are $n$ colonies. Moreover, a catastrophe is more likely to wipe out a smaller colony than a larger one. On the other hand, multiple colonies give multiple chances for survival and this turns out to be a critical advantage of the multiple colonies model over the single colony model.

\section{Proofs}

5.1. Auxiliary results. For $\eta_{t}$ being a $C C(\mathcal{G}, \lambda, p)$ process, we know that some colonization attempts will not succeed because the vertex on which the attempted colonization takes place is already occupied. This creates dependence between the number of new colonies created upon the collapse of different colonies. Because of 
this lack of independence, explicit probability computation seems impossible. In order to prove Theorem 3.1, we introduce a branching-like process which dominates $\eta_{t}$, in a certain sense, and for which explicit computations are possible. This process is denoted by $\xi_{t}$ and defined as follows.

\section{Auxiliary process $\xi_{t}$ :}

Each vertex of $\mathcal{G}$ might be empty or occupied by a number of colonies. Each colony starts from a single individual. The number of individuals in a colony at time $t$ is determined by a pure birth process of rate $\lambda$. Each colony is associated to a mean 1 exponential random variable. When the exponential clock rings for a colony it collapses and each individual, independently from everything else, survives with probability $p>0$ or dies with probability $1-p$. Each individual who survives tries to create a new colony at one of the nearest neighbor vertices picked at random. At every neighboring vertex we allow at most one new colony to be created.

Hence, in the process $\xi_{t}$ when a colony placed at vertex $x$ collapses, it is replaced by $0,1, .$. , or degree $(x)$ new colonies, each new colony on a distinct neighboring site of $x$. The process $\xi_{t}$ can be constructed graphically in a way similar to what we did for the colonization and collapse process. In many ways the process $\xi_{t}$ is similar to a branching random walk. In section 3 of Pemantle and Stacey (2001) a detailed graphical construction of a branching random walk was given. One can adapt their ideas to construct $\xi_{t}$.

Note that there is no bound on the number of colonies per site for $\xi_{t}$ but there is at most one colony per site for $\eta_{t}$. Observe that birth and collapse rates are the same for colonies in $\xi_{t}$ and $\eta_{t}$. To each colony created in process $\eta_{t}$ corresponds a colony created in the process $\xi_{t}$. But not every colony created in the process $\xi_{t}$ has its correspondent in the process $\eta_{t}$. Recall that in both processes every colony starts with a single individual. Techniques such as in Liggett (1985, Theorem 1.5 in chapter III) can be used to construct the processes $\xi_{t}$ and $\eta_{t}$ in the same probability space in such a way that, if they start with the same initial configuration, if there is a colony of size $i$ on a vertex $x$ for $\eta_{t}$ then there is at least one colony of size $i$ for $\xi_{t}$ on the same vertex $x$.

Lemma 5.1. Let $\mathcal{G}$ be a $m$-regular graph and $W_{m}(\lambda, p)$ the number of new colonies created by individuals of a collapsing colony in the process $\xi_{t}$ on $\mathcal{G}$. Then

(i) $\mu(m):=\mathbb{E}\left[W_{m}(\lambda, p)\right]=m-\frac{m}{\lambda} \sum_{k \geq 1} B\left(1+\frac{1}{\lambda}, k\right)\left(1-\frac{p}{m}\right)^{k}$.

(ii) $q_{\lambda}:=\mathbb{P}\left[W_{m}(\lambda, p)=m\right] \rightarrow 1$ as $\lambda \rightarrow \infty$.

Remark 5.2. Observe that for the process $\eta_{t}$ the probability that upon a collapse at vertex $x$ each one of its $m$ neighbors gets at least one colonization attempt is equal to $q_{\lambda}$.

Proof of Lemma 5.1: (i) Consider a colony at some vertex $x$ of $\mathcal{G}$. Let $Y$ be the number of individuals in the colony at collapse time. Then

$$
\mathbb{P}[Y=k]=\int_{0}^{\infty} e^{-t} e^{-\lambda t}\left(1-e^{-\lambda t}\right)^{k-1} d t=\frac{1}{\lambda} B\left(1+\frac{1}{\lambda}, k\right),
$$

where the last equality is obtained by the substitution $u=e^{-\lambda t}$ and the definition of the beta function. 
Enumerate each neighbor of vertex $x$ from 1 to $m$, then $W_{m}(\lambda, p)=\sum_{i=1}^{m} I_{i}$, where $I_{i}$ is the indicator function of the event $\{$ A new colony is created in the $i$-th neighbor of $x$. $\}$. Hence,

$$
\mathbb{E}\left[W_{m}(\lambda, p)\right]=\sum_{i=1}^{m} \mathbb{P}\left[I_{i}=1\right]=m \mathbb{P}\left[I_{1}=1\right] .
$$

Observe that

$$
\mathbb{P}\left[I_{1}=1 \mid Y=k\right]=1-\left(1-\frac{p}{m}\right)^{k}
$$

Therefore,

$$
\begin{aligned}
\mathbb{P}\left[I_{1}=1\right] & =\sum_{k=1}^{\infty}\left[1-\left(1-\frac{p}{m}\right)^{k}\right] \mathbb{P}[Y=k] \\
& =1-\frac{1}{\lambda} \sum_{k=1}^{\infty}\left(1-\frac{p}{m}\right)^{k} B\left(1+\frac{1}{\lambda}, k\right)
\end{aligned}
$$

where the last equality is obtained by (5.1). Substituting (5.3) in (5.2) we obtain the desired result.

(ii) Observe that

$$
\begin{aligned}
\mathbb{P}\left[W_{m}(\lambda, p)=m\right] & =\mathbb{P}\left[I_{1}=1, \ldots, I_{m}=1\right] \\
& =1-\mathbb{P}\left[I_{i}=0 \text { for some } i \in 1, \ldots m\right] \\
& \geq 1-\sum_{i=1}^{m} \mathbb{P}\left[I_{i}=0\right] \\
& =1-m \mathbb{P}\left[I_{1}=0\right] \\
& =1-\frac{m}{\lambda} \sum_{k=1}^{\infty}\left(1-\frac{p}{m}\right)^{k} B\left(1+\frac{1}{\lambda}, k\right) \\
& \geq 1-\frac{m}{\lambda} \sum_{k=1}^{\infty}\left(1-\frac{p}{m}\right)^{k} B(1, k)
\end{aligned}
$$

Letting $\lambda \rightarrow \infty$ in (5.4) we obtain the result.

\subsection{Proofs of main results.}

Proof of Theorem 3.1 (i): Consider $\xi_{t}$ starting with one colony at the origin and let $Z_{0}=1$. This colony we call the 0 -th generation. Upon collapse of that colony a random number of new colonies are created. Denote this random number by $Z_{1}$. These are the first generation colonies. Every first generation colony gives birth (at different random times) to a random number of new colonies. These new colonies are the second generation colonies and their total number is denoted by $Z_{2}$. More generally, let $n \geq 1$, if $Z_{n-1}=0$ then $Z_{n}=0$, if $Z_{n-1} \geq 1$ then $Z_{n}$ is the total number of colonies created by the previous generation colonies.

We claim that $Z_{n}, n=0,1, \ldots$ is a Galton-Watson process. This is so because the offsprings of different colonies in the process $\xi_{t}$ have the same distribution and are independent. 
The process $Z_{n}$ dies out if and only if $\mathbb{E}\left[Z_{1}\right] \leq 1$. From Lemma 5.1.(i) we know that $\mathbb{E}\left[Z_{1}\right]=\mu(m)$.

Observe that if the process $Z_{n}$ dies out, the same happens to $\xi_{t}$ and hence to $\eta_{t}$. It is easy to see that the proof works if the process starts from any finite number of colonies.

Proof of Theorem 3.1 (ii): The graphical construction of $\eta_{t}$ can be used to show that if two initial configurations are such that $\eta^{(0)}(x) \leq \eta^{(1)}(x)$ for every $x$ then for all $t \geq 0$ we have $\eta_{t}^{(0)}(x) \leq \eta_{t}^{(1)}(x)$. That is, the process $\eta_{t}$ is attractive. Hence, to prove the result we will prove local extinction for the process $\xi_{t}$ (which dominates $\eta_{t}$ ) starting with one individual at each vertex of $\mathbb{Z}^{d}$.

Fix a vertex $x$. If at time $t$ there exists a colony at vertex $x$ (for the process $\xi_{t}$ ) then it must descend from a colony present at time 0. Assume that the colony at $x$ descends from a colony at some site $y$. Let $Z_{n}(y)$ be the number of colonies at the $n$-th generation of the colony that started at $y$. The process $Z_{n}(y)$ has the same distribution as the process $Z_{n}$ defined above. In order for a descendent of $y$ to eventually reach $x$ the process $Z_{n}(y)$ must have survived for at least $d(x, y)$ generations. This is so because each generation gives birth only on nearest neighbors vertices. The process $Z_{n}(y)$ is a Galton-Watson process with $Z_{0}(y)=1$ and mean offspring $\mu=\mu(2 d)$.

Let $n=d(x, y)$, then

$$
\mathbb{P}\left(Z_{n}(y) \geq 1\right) \leq \mathbb{E}\left(Z_{n}(y)\right)=\mu^{n}=\mu^{d(x, y)}
$$

and

$$
\begin{gathered}
\sum_{y \in \mathbb{Z}^{d}} \mathbb{P}\left(Z_{n}(y) \geq 1\right) \leq \sum_{y \in \mathbb{Z}^{d}} \mu^{d(x, y)}= \\
\sum_{n \geq 1} \#\left\{x \in \mathbb{Z}^{d}: d(x, y)=n\right\} \mu^{n}=\sum_{n \geq 1}\left(\begin{array}{c}
n+d-1 \\
n
\end{array}\right) \mu^{n}<\infty,
\end{gathered}
$$

for $\mu<1$.

The Borel-Cantelli lemma shows that almost surely there are only finitely many $y$ 's such that descendents from $y$ eventually reach $x$. From $(i)$ we know that a process starting from a finite number of individuals dies out almost surely. Hence, after a finite random time there will be no colony at vertex $x$.

Proof of Theorem 3.1 (iii): The proof is analogous to (ii). In this case, $\mu=\mu(d+1)$ and

$$
\sum_{y \in \mathbb{T}^{d}} \mu^{d(x, y)}=\sum_{n \geq 1}(d+1) d^{n-1} \mu^{n}<\infty
$$

for $\mu<1 / d$.

Proof of Theorem 3.3: We first give the proof on the one dimensional lattice $\mathbb{Z}$.

We start by giving an informal construction of the process. We put a Poisson process with rate 1 at every site of $\mathbb{Z}$. All the Poisson processes are independent. At the Poisson process jump times the colony at the site collapses if there is a colony. If not, nothing happens. We start the process with finitely many colonies. Each colony starts with a single individual and is associated to a Yule process with birth rate $\lambda$. At collapse time, given that the colony at site $x$ has $n$ individuals we have a binomial random variable with parameters $(n, p)$. The binomial gives the 
number $k$ of potential survivors. If $k \geq 1$ then each survivor attempts to found a new colony on $x+1$ with probability $\frac{1}{2}$ or on $x-1$ also with probability $\frac{1}{2}$. The attempt succeeds on an empty site. We associate a new Yule process to a new colony, independently of everything else.

We use the block construction presented in Bramson and Durrett (1988). First some notation. For integers $m$ and $n$ we define

$$
\begin{gathered}
I=[-L, L] \quad I_{m}=2 m L+I, \\
B=(-4 L, 4 L) \times[0, T] \quad B_{m, n}=(2 m L, n T)+B,
\end{gathered}
$$

where

$$
T=\frac{5}{2} L
$$

and

$$
\mathbb{L}=\left\{(m, n) \in \mathbb{Z}^{2}: m+n \text { is even }\right\} .
$$

We say that the interval $I_{m}$ is half-full if at least every other site of $I_{m}$ is occupied. That is, the gap between two occupied sites in $I_{m}$ is at most one site.

We declare $(m, n) \in \mathbb{L}$ wet if starting with $I_{m}$ half-full at time $n T$ then $I_{m-1}$ and $I_{m+1}$ are also half-full at time $(n+1) T$. Moreover, we want the last event to happen using only the Poisson and Yule processes inside the box $B_{m, n}$. That is, we consider the process restricted to $B_{m, n}$.

We are going to show that for any $\epsilon>0$ there are $\lambda, L$ and $T$ so that for any $(m, n) \in \mathbb{L}$

$$
P((m, n) \text { is wet }) \geq 1-\epsilon .
$$

By translation invariance it is enough to prove this for $(m, n)=(0,0)$. The proof has two steps.

- Let $E$ be the event that every collapse in the finite space-time box $B$ is followed by at least one attempted colonization on the left and one on the right of the collapsed site. We claim that for every $\epsilon>0$, we can pick $L, T$ and $\lambda>0$ large enough so that $P(E) \geq 1-\epsilon$. We now give the outline of why this is true. Since collapse times are given by rate one Poisson processes on each site of $B$ the total number of collapses inside $B$ is bounded above by a Poisson distribution with rate $(8 L+1) T$. Hence, with high probability there are less than $2(8 L+1) T$ collapses inside $B$ for $L$ large enough. We also take $\lambda$ large enough so that at every collapsing time the colony will have so many individuals that attempted colonizations to the left and right will be almost certain (see Lemma 5.1.(ii)). Since the number of collapses can be bounded with high probability the probability of the event $E$ can be made arbitrarily close to 1 .

- At time 0 we start the process with the interval $I$ half-full. Let $r_{t}$ and $\ell_{t}$ be respectively the leftmost and rightmost occupied sites at time $t \geq 0$. Conditioned on the event $E$ it is easy to see that the interval $\left[\ell_{t}, r_{t}\right]$ is half-full at any time $t \leq T$. Observe also that conditioned on $E$, every time there is a collapse at $r_{t}$ then $r_{t}$ jumps to $r_{t}+1$. Since the number of collapses at $r_{t}$ is a Poisson process with rate 1 we have that $\frac{r_{t}}{t}$ converges to 1 . Hence, for $T=\frac{5}{2} L$ we have that $r_{T}$ belongs to $(3 L, 4 L)$ with a probability arbitrarily close to 1 provided $L$ is large enough. A symmetric argument shows that $\ell_{T}$ belongs to $(-4 L,-3 L)$. Since the interval $\left[\ell_{T}, r_{T}\right]$ contains $I_{-1}$ and $I_{1}$, both of these intervals are half-full. Hence, for any $\epsilon>0$ we can pick $L$ and $\lambda$ large enough so that $P((0,0))$ is wet $) \geq 1-\epsilon$. 
The preceding construction gives a coupling between our colonization and collapse model and an oriented percolation model on $\mathbb{L}$. The oriented percolation model is 1-dependent and it is well known that for $\epsilon>0$ small enough $(0,0)$ will be in an infinite wet cluster which contains infinitely many vertices like $(0,2 n)$, see Durrett (1984). That fact corresponds, by the coupling, to local survival in the colonization and collapse model. Note that the proof was done for the process restricted to the boxes $B_{m, n}$. However, if this model survives then so does the unrestricted model. This is so because the model is attractive and more births can only help survival.

Consider now the model on $\mathbb{Z}^{d}$ with $d \geq 2$ and parameters $(p, \lambda)$. Fix a line on $\mathbb{Z}^{d}$. It is easy to see that we may couple the process on $\mathbb{Z}^{d}$ with the process on $\mathbb{Z}$ with parameters $(p / d, \lambda)$ in such a way that the process on $\mathbb{Z}$ has less individuals per site than the process on the embedded line of $\mathbb{Z}^{d}$. This is so because births on $\mathbb{Z}$ will correspond to births on the embedded line and collapses are coupled. By Theorem 3.3 we can find $\lambda$ large enough so that the process on $\mathbb{Z}$ with parameters $(p / d, \lambda)$ survives locally. Hence, for the same $\lambda$ the process on $\mathbb{Z}^{d}$ with parameters $(p, \lambda)$ survives locally.

For $\mathbb{T}^{d}$ the local survival follows analogously as in $\mathbb{Z}^{d}$, observing that $\mathbb{Z}$ is embedded in $\mathbb{T}^{d}$.

Remark 5.3. Our argument shows that both critical values, $\lambda_{c}\left(p, \mathbb{Z}^{d}\right)$ and $\lambda_{c}\left(p, \mathbb{T}^{d}\right)$, decrease with $d$. The more difficult issue is whether the critical value is strictly decreasing. We conjecture it is but this is a hard question even for the contact process, see Liggett (1999).

\section{Acknowledgements}

Thanks are due to the anonymous referees for their careful reading, criticism and suggestions which helped us to considerably improve the paper.

\section{References}

M. Bramson and R. Durrett. A simple proof of the stability criterion of Gray and Griffeath. Probab. Theory Related Fields 80 (2), 293-298 (1988). MR968822.

P. J. Brockwell. The extinction time of a general birth and death process with catastrophes. J. Appl. Probab. 23 (4), 851-858 (1986). MR867182.

R. Durrett. Oriented percolation in two dimensions. Ann. Probab. 12 (4), 999-1040 (1984). MR757768.

I. Hanski. Metapopulation Ecology. Oxford Univ. Press, Oxford. (1999). ISBN 9780198540656.

T. E. Harris. Nearest-neighbor Markov interaction processes on multidimensional lattices. Advances in Math. 9, 66-89 (1972). MR0307392.

S. Kapodistria, T. Phung-Duc and J. Resing. Linear birth/immigration-death process with binomial catastrophes. Probab. Engrg. Inform. Sci. 30 (1), 79-111 (2016). MR3436782.

T. M. Liggett. Interacting particle systems, volume 276 of Grundlehren der Mathematischen Wissenschaften [Fundamental Principles of Mathematical Sciences]. Springer-Verlag, New York (1985). ISBN 0-387-96069-4. MR776231. 
T. M. Liggett. Stochastic interacting systems: contact, voter and exclusion processes, volume 324 of Grundlehren der Mathematischen Wissenschaften [Fundamental Principles of Mathematical Sciences]. Springer-Verlag, Berlin (1999). ISBN 3-540-65995-1. MR1717346.

Y. L. Luke. The special functions and their approximations, Vol. I. Mathematics in Science and Engineering, Vol. 53. Academic Press, New York-London (1969). MR0241700.

R. Pemantle and A. M. Stacey. The branching random walk and contact process on Galton-Watson and nonhomogeneous trees. Ann. Probab. 29 (4), 1563-1590 (2001). MR1880232.

R. B. Schinazi. Does random dispersion help survival? J. Stat. Phys. 159 (1), 101-107 (2015). MR3320953. 